\title{
El Poder Legislativo y los partidos políticos
}

\subsection{Breve esbozo histórico y caracterización del poder legislativo}

Para una mejor compresión antes de abordar el tema es necesario, definir el térnnino Parlamento. Parlamento tiene su origen de la palabra francesa parler, que en español significa, parlar, es decir, hablar o conversar unos con otros. Según el Diccionario de la Real Academia de la Lengua Españolal, existen siete significados de la palabra Parlamento:

1. Asamblea de los grandes del reino, que bajo los primeros reyes de Francia se convocaba para tratar negocios importantes.

2. Cada uno de los tribunales superiores de justicia que en Francia tenían además atribuciones políticas y de policía.

3. La Cámara de los Lores y la de los Comunes en Inglaterra.

4. Por extensión Asamblea legislativa.

5. Razonamiento u oración que se dirigfa a un congreso o junta.

6. Entre actores, relación larga en verso o prosa

7. Acción de parlamentar.

El Parlamento ha evolucionado, por ejemplo, en sus funciones, composición y estructura, éstos cambios han ocurrido de acuerdo con el contexto particular de alguna zona geográfica determinada, en donde se haya creado y desarrollado históricamente un Estado.

Cada Estado tiene un Parlamento diferenle y peculiar, que puede ser considerado en última instancia como el resultado de los cambios o modificaciones ocurridos en el tiempo en una sociedad y que pudieron ser influidos directa o indirectamente por sucesos de carácter nacional, regionales o internacionales. 
El origen del Parlamento se encuentra en Europa, en donde los reyes convocaban y presidían el Parlamento, ésta institución cumplfa sobre todo funciones judiciales, económicas y presupuestarias.

En el siglo XIV, los órganos parlamentarios, despojados de sus funciones judiciales, incorporaron a otros representantes de sectores sociales, plebeyos, urbanos, a la gentry en Inglaterra, a lo que en Francia se llama «estado llano" y en España al tercer brazo: burgueses, propietarios, etc., los meliores. terrae. Con esta representación, los Parlamentos estamentales entran en complejas relaciones entre sí y con el Monarca. Así, en Inglaterra, comienza a imponerse la práctica de que los nobles temporales y espirituales se reúnan aparte (lo que da origen a la Cámara de los Lores) en tanto que el estamento llano lo hace por otra (lo que da origen a la Cámara de los Comunes); igual sucederá con los Estados Generales en Francia y la costumbre se romperá por primera vez, cuando los tres Estados deciden celebrar sesiones conjuntas en 1789: con anterioridad, el estado llano había impuesto la costumbre de presentar al Monarca los "cahiers de doléances, en los cuales se contenian sus reivindicaciones, cuyo logro sollan vincular a la aprobación de los impuestos por el Rey2.

El origen del Parlamento no se puede interpretar como un intento de instaurar sistemas políticos democráticos, sin embargo, con el aparecimiento y forlalecimiento de la burguesfa en el siglo XVI; con los efectos económicos, políticos y sociales del advenimiento del capitalismo, en el siglo XVIII; los efectos directos y mediatos de las Primera y Segunda guerras mundiales, en el siglo XX, los parlamentos fueron objetos de cambios considerables, hasta concebirse hoy en día como los pricipales órganos del Estado.

Al Parlamento se le designa indistintamente con varios nombres, no obstante, en el desarrollo del presente trabajo se empleará el de Asamblea Legislativa.

Entre los sinónimos de Asamblea Legislativa encontramos los siguientes (ver cuadro $\mathrm{N}^{\circ} .1$ ): 
CUADRO No.1

ALGUNOS SINÓNIMOS DE ASAMBLEA LEGISLATIVA POR PAfS

\begin{tabular}{ll}
\hline ASAMBLEA LEGISLATIVA & PAf́s \\
\hline Asamblea Federal & Suiza \\
Asamblea Popular & Albania \\
Cámara de Representantes & Indonesia \\
Congreso Federal & EE.UU. \\
Congreso Popular Nacional & China \\
Consejo Supremo & Letonia \\
Cortes & España \\
Dieta & Japón \\
Gran Asamblea & Rumania \\
Knesset & Israel \\
Parlamento & Inglaterra \\
Estados Generales & Parses Bajos \\
Consejo Grande y General & San Marino \\
\hline
\end{tabular}

Las Asambleas Legislativas pueden ser unicamerales (monocameralismo), constituidas por una sola cámara o, bicamerales (bicameralismo), formadas por dos cámaras.

El sistema unićameral fue el primero en surgir, luego éste dio origen al bicameral y tuvo su génesis en Inglaterra, cuando los commons o baja nobleza formaron su propia cámara, la Cámara de los Comunes, como resultado de la decisión que tomó la aristocracia o los lores de tener reuniones en la Cámara de los Lores, separadas de los commons. Con esta escisión cada cámara representaba intereses distintos y en muchos casos contrapuestos, debido, entre otros, por las diferencias sociales y económicas de los miembros que componían cada una de las cámaras.

El sistema legislativo inglés se extendió por casi toda Europa, valga decir que los países lo adoptaron y lo modificaron, acorde con las particularidades de cada Estado, una vez que formó parte del sistema político de los países, éste evolucionó y desarrollo en la medida que se transformaron las sociedades europeas y actualmente es el sisterna predominante en Europa.

Montesquieu (...) propuso que se dividiera en dos Cámaras que, siguiendo el ejemplo inglés, representaran a la sociedad de acuerdo con sus partes: la baja para el pueblo y la alta para los propietarios3. 
Montesquieu hacía descansar este último control en un principio social, más que polftico y propio del Estado. Esla aladura aristocrática se rompería con la aparición de los federalistas estadounidenses que reformularon la división del Congreso para asignar a la Cámara Baja la representación ciudadana y al Senado la de los Estados Federados4.

Hoy en día, en los países de la comunidad internacional, los poderes legislativos ya no son un escenario en donde se reproduce la división de clases de la sociedad, en donde por un lado están representados los propietarios y en el otro los trabajadores. Esto no significa que las cámaras no puedan estar constituidas por partidos políticos que encarnen expresiones de clase y posiciones antinacionales.

En los sistemas bicamerales, las cámaras han logrado alcanzar un equilibrio entre ellas, y se han convertido en complementarias.

Las cámaras, de los poderes legislativos bicamerales tienen diferentes nombres, según el pars (ver cuadro No.2).

CUADRO No.2

ASAMBLEAS LEGISLATIVAS BICAMERALES

\begin{tabular}{lll}
\hline PAÍS & PRIMERA CÁMARA & SEGUNDA CÁMARA \\
\hline Botswana & Cámara de Jefes & Asamblea Nacional \\
EE.UU. & Senado & Cámara de Representantes \\
Eslovenia & Consejo de Estado & Asamblea del Estado \\
España & Senado & Congreso \\
India & Consejo de los Estados & Cámara del Pueblo \\
Paraguay & Senado & Cámara de Diputados \\
Suiza & Consejo de los Estados & Consejo Nacional \\
\hline
\end{tabular}

El poder legislativo nació primero que la teoría, fue el resultado de los sucesos históricos el que lo concibió y, se puede decir, que por los hechos positivos que causó u originó en la vida de los pueblos, fue lo que le concedió prácticamente validez universal. "Lo que Inglaterra fue construyendo orgánicamente, sin razonarlo casi, a medida que las circunstancias lo aconsejaban, vino a ser teorizado en el Continente"s.

Sin embargo, hasta la fecha no se ha elaborado una teoría que se constituya en paradigma ideal para ser copiado por cualquier Estado. En este orden cada Asamblea Legislativa es sui generis y se constituye en la secuela directa del proceso y nivel político, social, económico, cultural, técnico y científico, etc., entre otros, alcanzado por cada pals. 
En este sentido, el funcionamiento, composición y funciones de la Asamblea Legislativa estarán en proporción directa con el desarrollo del país respectivo, en otras palabras, el estado en que se encuentre cada Asamblea Legislativa es el reflejo del nivel de crecimiento y desarrollo alcanzado en diversas áreas y sectores prioritarios de la sociedad, lo que incluye obviamente el nivel de democracia logrado.

\subsubsection{Sistema unicameral y bicameral}

La composición y el número de miembros de ambas cámaras varía de pals en país, no solamente tomando como parámetros las peculiaridades históricas de cada uno, sino también la densidad de la población, la extensión territorial y la división política-administrativa del Estado.

CUADRO No.3

ASAMBLEAS LEGISLATIVAS BICAMERALES*

POBLACIÓN, ÁREA(Km²), MIEMBROS Y PERÍODOS

DE LA LEGISLATURAS

\begin{tabular}{|c|c|c|c|c|c|c|}
\hline \multirow[b]{2}{*}{ PAIS } & \multicolumn{3}{|c|}{ PRIMERA CÁMARA } & \multicolumn{3}{|c|}{ SEGUNDA CAMARA } \\
\hline & Población & Área $\left(\mathrm{Km}^{2}\right)$ & Miembros & Legislatura & Miembros & Legislatura \\
\hline Bolivia & 7.065 .000 & 1.098 .581 & 27 & 4 años & 130 & 4 años \\
\hline EE.UU. & 258.233 .000 & 9.372 .614 & 100 & 6 anios & 435 & 2 años \\
\hline India & 896.567 .000 & 3.287 .590 & 245 & 6 años & 545 & 5 años \\
\hline Paraguay"• & 4.643 .000 & 406.572 & 45 & 5 años & 80 & 5 años \\
\hline Reino Unido & $57,826.00$ & 244.100 & 1,222 & $\begin{array}{l}\text { hereditarios } \\
\text { y vitalicios }\end{array}$ & 650 & 5 años \\
\hline
\end{tabular}

- Se emplea Primera Cámara y Segunda Cámara para referirse a sus respectivos homólogos en los países mencionados en el cuadro.

** Elegidos por un período de cinco años y puede ser disuelta por el ejecutivo antes del quinquenio.

Fuente: Datos obtenidos del Almanaque Mundial 1996, Virginia, Editorial Televisa, 1995.

En algunos países que tienen división política federal optan por el sistema legislativo bicameral, en estas situaciones la Primera Cámara (el Senado), es donde se da la representación territorial, puesto que, por lo general es formado por uno, dos o más representantes de los Estados o Provincias del país y son elegidos ya sea por medio del sufragio universal, por el representante del Rey 
(gobernador), el Rey, o pueden ser personas que han adquirido el derecho por herencia familiar, como en el caso del Reino Unido.

En los sistemas bicamerales de los Estados Federados, además de existir las dos cámaras, por lo general localizadas en la capital del Estado, coexisten de la misma forma igual número de Asambleas Legislativas como provincias o Estados posea el pars, por ejemplo, en Eslados Unidos existe el Congreso Federal, pero además hay cincuenta Asambleas Legislativas, es decir, una por cada Estado6.

El período como senadores puede ser por cuatro o seis años, por toda la vida como en el Reino Unido o hasta cuando el senador cumpla setenta y cinco años de edad, como el caso de Canadá7.

En la Segunda Cámara (Cámaras de Representantes), es donde se da la representación del pueblo y está constituida, por lo general, por diputados que son elegidos proporcionalmente con la población que tiene cada uno de los respectivos Estados o Provincias del paŕs, de tal forma que habrá más diputados de una provincia que de otra, puesto que el número de ellos lo determina la densidad de la poblacion.

Asimismo, se cuenta otro sistema parlamentario denominado tricameral o multicameral, pero realmente éste no llega a constituirse en una institución formada por tres o más cámaras equilibradas entre ellas, sino que lo que predomina es el bicameral, lo que sucede es que se crean algunos comités, consejos o comisiones legislativas especiales a las que llaman cámaras, que adquieren cierta importancia al interior de la Asamblea Legislativa, pero no igual ni mayor que las dos cámaras predominantes.

Francia y muchos países africanos han pasado por la experiencia de incluir una tercera cámara representante de intereses funcionales, en sus sistemas bicamerales basados en la representación territorial. Estas cámaras funcionales son consejos económicos y sociales que juegan un papel puramente consultivo que no justifica que el sistema pueda ser considerado como verdaderamente tricameral8.

En cuanto al sistema unicameral y bicameral, la doctrina todavía no se ha puesto de acuerdo sobre cuál de los dos es el mejor, puesto que no es la teoría la que define el buen funcionamiento del poder legislativo o de una legislación, sino los resultados positivos y el rol destacado o determinante que éste órgano desempeñe en la construcción, reconstrucción o proceso de consolidación de la democracia de algún pars. Sin embargo, resulta interesante hacer notar que la tendencia observada en los países del mundo es el acentuado predominio del sistema unicameral, "la proporción es de casi tres conira uno"9.

Por un lado se esgrimen argumentos que tratan de justificar la existencia del sistema unicameral, entre los cuales encontramos los siguientes: 
- La soberanía es indivisible y por lo tanto no se tiene que dividir en dos o en más partes el Poder Legislativo. Juan Jacobo Rousseau, sostenía que: "la soberanía es inalienable, es indivisible" 10.

- El sistema bicameral es un remanente ya superado de las épocas donde el reinado era el predominante.

- Con el sistema bicameral se duplica el trabajo y el tiempo para resolver o tratar las mismas leyes, piezas de correspondencias, etc., además que representa un doble gasto presupuestario del pals.

- Con el bicameralismo se producen competencias y rivalidades innecesarias entre ambas cámaras, que afectan no sólo al buen funcionamiento del poder legislativo sino, también, al del sistema político y, en definitiva, al de la población.

- El unicameralismo permite más agilidad y flexibilidad al Ejecutivo, puesto que el presidente del pafs no tiene que dialogar, negociar o enfrentarse a dos cámaras.

- El tradicionalismo aristocrático de la nobleza y sus prerrogativas ya están superadas por la historia como para que exista una Cámara Alta.

Por otra parte, también encontramos argumentos que sostienen la necesidad de la existencia de las dos Cámaras Legislativas, por ejemplo:

- Las resoluciones, dictámenes, leyes, decretos, etc., serán ampliamente y mejor analizados, en ese sentido, los errores que cometan los diputados de la primera cámara serán rectificados o corregidos por los de la segunda.

- La existencia de ambas cámaras es saludable para garantizar de forma idónea el equilibrio del poder, la autonomfa e independencia del Legislativo en relación con los Poderes Ejecutivo y Judicial.

- Es bueno la existencia de un contrapeso y freno a la primera cámara, porque ésta puede devenir en autoritaria e impositiva sobre el Ejecutivo y sobre las demás instituciones públicas y privadas del pals.

- Con el sistema bicameral se contribuye a estimular la carrera parlamentaria, puesto que el diputado de la primera cámara tratará de ascender a la segunda, lo que le permitiría lograr un aumento salarial, nuevas inmunidades y prerrogativas que se desprenderían de su nuevo rol, a la vez, mejorar su status frente a la clase y sociedad política, asimismo, frente a la población civil.

- Se produce una división racional del trabajo entre ambas cámaras, la cual redunda en agilidad, eficiencia y funcionalidad del Poder Legislativo para abordar los tópicos y documentos que ingresan a la Asamblea Legislativa. 
- La versión bicameral es la clásica de origen inglesa y es la que debe predominar, ya que la unicameral es una degeneración o desvirtualización del bicameralismo.

Para el caso de los países de Centro América, el Poder Legislativo es unicameral, en El Salvador la Asamblea Legislativa está constituida por 84 diputados elegidos por sufragio universal por un perfodo de tres años.

\section{CUADRO No.4 \\ LAS ASAMBLEAS LEGISLATIVAS DE LOS PAISES DE CENTRO AMÉRICA}

\begin{tabular}{lrccc}
\hline País & $\begin{array}{c}\text { Población } \\
\text { (millones) }\end{array}$ & $\begin{array}{c}\text { Área }\left(\mathbf{K m}^{2}\right) \\
(\mathrm{miles})\end{array}$ & $\begin{array}{c}\text { Número de } \\
\text { Diputados }\end{array}$ & $\begin{array}{c}\text { Duración } \\
\text { del período }\end{array}$ \\
\hline Guatemala & 10.029 .000 & 108.889 & 80 & 4 años \\
Honduras & 5.595 .000 & 112.088 & 128 & 4 años \\
Nicaragua & 4.265 .000 & 130.700 & 92 & 6 años \\
Costa Rica & 3.199 .000 & 51.100 & 57 & 4 años \\
El Salvador & 5.517 .000 & 21.040 .79 & 84 & 3 años \\
\hline
\end{tabular}

- Estimación media de la población en los años 1992 y 1993.

Fuente: Datos obtenidos del Almanaque Mundial 1996, Virginia, Editorial Televisa, 1995.

\subsubsection{Clasificación del sistema de gobierno legislativo}

En la caracterización del sistema legislativo, partimos del hecho de que no existe uno que se pueda tomar como modelo ideal' ni realizar la distinción en la medida de la aproximación o lejanla de rasgos comunes de determinado sistema.

- No existen dos sistemas iguales', los hay similares, lo que significa que cada uno tiene sus propias particularidades, podemos afirmar que cada sistema responde a una historia, geograffa, economía, politica, etc., especffica del pals.

La caracterización se hará a continuación de manera general y no exhaustiva de tal forma que se tomarán algunos parámetros similares, pero obviamente se dará el caso de que algunas características no se aplican a algún sistema, ya sea presidencialista o parlamentario. No obstante, el denominador común entre ambos sistemas es que son de carácter representativo., 


\subsubsection{Sistema de gobierno presidencialista -}

- El presidencialismo fue establecido por primera vez en los Estados Unidos de América por los federalistas mediante la Constitución estadounidense del 17 de septiembre de 1787, posteriormente fue adoptado con las modificaciones del caso por la mayoría de los paŕses de América Latina y, en la actualidad ha llcgado a ser el sistema predominante.

- Históricamente, el sistema presidencialista es más antiguo que el parlamentario/Al mismo pertenecen las monarqulas constitucionales en las que la constitución establece que el gobierno depende únicamente de la confianza del monarca, mientras que el parlamento participa como instancia legislativa en el proceso político.'La democratización del sistema presidencialista significó la abolición de la monarquía, porque el sistema de gobierno presidencialista y la democracia son compatibles en el marco de la repúblicalb

- En el presidencialismo existe una separación, equilibrio, autonomía e independencia relativa entre los poderes Legislativo, Ejeculivo y Judicialoy, la relación que existe entre ellos es de coordinación, por lo que ésta separación de poderes es tipificada como rígida.

'En éste sistema de gobierno el presidente está investido con la función de jefe del Ejecutivo o del gobierno, del Estado, comandante en jefe de las Fuerzas Armadas y dirige la diplomacia o relaciones internacionales del país 12 .

- El presidente puede nombrar de manera discrecional a los ministros del gobierno.o secretarios de Estadar éstos últimos no son responsables políticamente ante la Asamblea; de la misma manera nombra a cierto personal clave en la administración pública.

El presidente y el vice presidente, al igual que los diputados que conforman la Asamblea Legislativa, son elegidos por el cuerpo electoral del país, a través del sufragio universal para un perfodo determinado, por lo que tanto el presidente y el vice presidente como la Asamblea Legislativa están investidos con la representatividad que emana de los ciudadanos y, por lo tanto, s6lo responden de su gestion ante el pueblo.

En la elección de los diputados tienen la oportunidad de llegar a la Asamblea, incluso hasta un sólo diputado que rêpresente a un partido político pequeño.

En la elección del presidente y vicepresidente, el partido político o coalición que salga victorioso de las elecciones, llega solo al Ejecutivo, es decir, es "suma cero", el vencedor se lleva todo el "premio", el cual es indivisible y los perdedores quedan absolutamente excluidos del Ejecutivo y de la dirección de la administración pública. No obstante, esta situación no es impedimento para formar coaliciones políticas en el seno de la Asamblea Legislativa que apoyen o respal- 
den al Ejecutivo en la formación de políticas públicas.

El presidente y la Asamblea Legislativa se encuentran en un plano de igualdad y equilibrio mutuo. El presidente puede remover o destituir a miembros de su gabinete de ministros, ya que éstos son responsables de su gestión sólo ante él. El presidente no puede disolver a la Asamblea cuando lo considere necesario y convocar a nuevas elecciones, ni la Asamblea destituir al presidente (salvo en casos excepcionales contemplados en las Constituciones) y disolver su gobiemo por medio de un volo de censura o desconfianza.

Esta situación ha sido excusa que han utilizado los militares en América Latina para dar golpes de Estado cuando ellos lo han estimado conveniente, precisamente por no existir un mecanismo que sirva de "fusible" o "válvula de escape" en el sistema político para resolver las crisis que se producen y activar mecanismos que las diriman.

Sin embargo, la historia enseña que los militares cuando dan los cuartelazos o golpes de Estado no es precisamente para resolver crisis políticas o económicas, sino para crearlas y agravarlas. Aquí no cabe la duda si el sistema presidencialista es el disfuncional, sino la perjudicial e innecesaria existencia de las Fuerzas Armadas en los países de América Latina.

\subsubsection{Sistema de gobierno parlamentario}

- "El parlamentarismo de Inglaterra se ha ido configurando paulatinamente, a partir de la segunda mitad del siglo XVI, se extendió primero a Francia"13, que sirvio de puerta de entrada al continente europeo y fue el país donde se perfecciono.

¿Es de hacer notar que en el actual parlamentarismo los tres poderes del Estado desarrollan relaciones de integracion entre ellos, por lo que la división es calificada como flexible, situación que facilita la formación de alianzas entre los partidos políticos representados en el Parlamento.

'El poder Ejeculivo en la mayoría de los casos tiene una organización dual, está dividido entre el jefe de Estado y el jefe del gobiemo. .

El jefe de Estado no posee un poder fuerte ni propio, pero desarrolla un papel simbólico y de influencia psicosocial en la población, que podramos definir como esencial para mantener la integración nacional.

'El primer ministro es el jefe del gobierno o del Ejecutivols, desempeña las funciones ejecutivas, preside y dirige el gabinete ministerial.

-El jefe del gobierno y los miembros del gabinete son seleccionados por el Parlamento, por lo que individual y colectivamente dependen del beneplácito y confianza del Poder L-egislativo. 
El Ejecutivo, incluido obviamente el primer ministro, tiene responsabilidad política ante el Parlamento, lo que significa, que el parlamento puede disolver todo el gabinete o sólo destituir a los ministros y dejar al primer ministro; mediante un voto de censura o de desconfianza.

Con posterioridad a la II Guerra Mundial, los sistemas parlamentarios han introducido un mecanismo tendente a evitar la inestabilidad de los gabinetes: el voto de censura constructiva. Este mecanismo, instaurado por primera vez en la Ley Fundamental de Bonn 15 y adoptado, entre otras, por la Constitución Española de 197816, exige que la moción de censura de un gabinete esté acompañada con la propuesta de un candidato para ocupar la jefatura de gabinete17.

Sin embargo, el gobiemo tiene la misma prerrogativa, puede disolver una o ambas cámaras del Parlamento y convocar inmediatamente a elecciones; de esta forma es la ciudadanía la que con su voto tratará en cierta medida de dirimir el conflicto que dio origen a la disolución. Por tal razón, el gobierno al elaborar su agenda nacional e internacional sabe de antemano que debe ser del agrado y aprobación del Parlamento, porque de lo contrario podría derivar en conflicto entre ambos poderes.

'La disolución de una o ambas cámaras también puede realizarla el jefe de gobiemo y es decir, para saber si la población está o no de acuerdo con determinada decisión del Ejecutivo, la cual es de trascendencia para la nación, por ejemplo: "En 1982, ante el conflicto de las islas Malvinas, la Primera Ministra Margaret Thacher, disolvió la cámara y llamó a nuevas elecciones anticipadamente, de esa manera obtuvo clara mayoría parlamentaria" 18.

En resumen, para Linz la mejor manera de reseñar las diferencias básicas entre el presidencialismo y el parlamentarismo consiste en destacar que mientras el parlamentarismo le otorga al proceso político flexibilidad, el presidencialismo lo toma rígido. A la probable crítica a esta concepción por parte de los defensores del presidencialismo, diciendo que la rigidez es una ventaja, pues salvaguarda al proceso político de la incertidumbre e inestabilidad propias del parlamentarismo, contesta afirmando que aunque la necesidad de autoridad y predicción parecería favorecer al presidencialismo, existen circunstancias inesperadas como puede ser la muerte del titular del Ejecutivo o la toma de decisiones erróneas, que convierten al gobierno presidencial en menos predecible y a menudo más débil que el de un Primer Ministro19.

Los problemas en el funcionamiento del presidencialismo latinoamericano, según Nohlen20, no deben conducir a una exigencia por transformar radicalmente el régimen político, esto es instaurar el parlamentarismo, sino más bien producir modificaciones que tengan en cuenta las circunstancias particulares de cada país21. 
Por otra parte, en ciertos palses se han desarrollado últimamente sistemas legislativos denominados mixtos o semipresidenciales, por estar formados con algunos elementos de los dos sistemas, sin embargo, en la realidad tiende invariablemente a predominar el sistema presidencial o parlamentario.

El semipresidencialismo es un hrbrido y un intento ecléctico que no se puede situar como un sisterna intermedio entre ambos y que sea mejor a cualquiera de los dos que le dieron origen.

Por otra parte, definir las caracteristicas fundamentales entre el presidencialismo y el parlamentarismo es difícil. Por esta razón hay inclinación a tomar de manera muy simplista como paradigma del presidencialismo al Congreso Federal de los Estados Unidos y como paradigma del parlamentarismo al Parlamento del Reino Unido, asimismo, dentro de esta lógica, como modelo del semipresidencialismo al sistema legislativo de Francia.

Entre los países que tienen sistema de gobierno semipresidencial podemos mencionar los siguientes: Argentina, Austria, Bolivia, Finlandia, Irlanda, Islandia, Perú y Portugal. En este mismo orden de clasificación de sistemas de gobiernos, el de Suiza es el único que no se puede tipicar como presidencialista, parlamentario o mixto, por lo que es llamado directorial o colegial. El poder político en este caso no se encuentra personificado en el jefe de Estado o de gobierno sino que reside en el directorio y no existe, entre otros, el voto de censura.

Carlos Sabino, profesor titular de la Universidad Central de Venezuela, sostiene para el caso de Venezuela que:

Como un modo de reducir el presidencialismo predominante y con el objeto, además, de distanciar a la figura del presidente de los movimientos coyunturales de opinión, se ha propuesto la creación del cargo de Primer Ministro. Se crearía así un régimen semi-presidencial, en algún modo semejante al francés, que harfa al Primer Ministro responsable ante el Parlamento pero dejando al Presidente como figura suprema del Estado y del gobierno. Quienes defienden esta modalidad de gobierno señalan que olorga, a la vez, las ventajas del presidencialismo y del parlamentarismo, dando mayor flexibilidad a la obra de gobierno, haciendo más responsable al Ejecutivo frente al Congreso y facilitando una válvula de escape para superar las crisis políticas. Quienes se oponen destacan, por otra parte, que el nuevo cargo no podría adquirir real significación dentro de nuestra tradición polftica, convirtiéndose simplemente en un ministro más, y que en nada reducirfa el presidencialismo vigente22.

\subsection{Representatividad parlamentaria popular o partidista}

En El Salvador no ha existido una acumulación democrática, como tampoco 
un régimen de partidos políticos que se haya desenvuelto en un contexto democrático; el autoritarismo siempre ha estado presente con uniforme militar o con la versión civil, como la actual.

A la población le ha sido sistemáticamente impedido a través de la historia el desartollo de una tradición partidista. Al ciudadano prácticamente le han vedado encuadrarse dentro de algún partido político que no sea de derecha, las simpatías o militancia por un partido progresista o de izquierda lo ha llevado a sufrir represalias por parte de las autoridades policiales, militares y hasta laborales, etc.

Actualmente, el salvadoreño que milite en otro partido que no sea de derecha o especlficamente en el Partido Alianza Republicana Nacionalista (ARENA), tiene serios problemas porque su partido no se presentará a las elecciones en las mismas condiciones que el partido de la oligarqufa y de los militares, porque el sistema jurídico y en particular el electoral sólo favorece a la derecha.

Esta situación ayuda a mantener y a prolongar la permanencia de la derecha en el poder político y a concentrar cada vez más la riqueza de la nación en manos de una minoría privilegiada; pero también hay otros factores que favorecen a ARENA. Por ejemplo:

- Debilidad organizacional y falta de un programa o plan nacional coherente de los partidos políticos de oposición.

- Falta de democratización, alternabilidad en la dirigencia y modernización de los actuales sindicatos, gremios, asociaciones y, en general, del movimiento social, cuya mayoría demuestra tener, tanto al interior de sus organizaciones como en su entorno externo, un notorio desconocimiento o violación de las reglas y valores elementales de la ética.

- El divisionismo al interior de los otros partidos políticos, debido a las ambiciones personales de poder y de protagismo.

- La izquierda y, fundamentalmente, el Partido Frente Farabundo Martí para la Liberación Nacional (FMLN), está tratando de alejarse de su posición histórica, polílica e ideológica de izquierda, para tratar de ubicarse como centro23.

- Los partidos progresistas no recuperan o rehabilitan la identidad nacional y ni crean "utopías" que le ofrezcan a la población esperanzas en el futuro.

- Falta de capacidad propositiva de los demás partidos polf́ticos ante los principales problemas que afectan a la población, particularmente ante el desempleo, la pobreza, bajos salarios, delincuencia, etc.

- Los otros partidos de derecha o centro derecha que siempre terminan vendiéndose o entregándose a la defensa y protección de los poderosos intereses económicos y financieros. 
- Esta situación de los partidos polfticos, por ejemplo, imposibilita comenzar a caminar por el sendero de la democracia y abandonar el del autoritarismo.

Por otro lado, las deficiencias del Tribunal Supremo Electoral (TSE) contribuyen grandemente a mantener y a consolidar el autoritarismo, asimismo, a brindarle estabilidad en su desarrollo y evolución.

En este mismo orden, los partidos de oposición, al igual que el Estado, necesi$\checkmark$ tan modernización, pero modemizar a los partidos progresistas no quiere decir convertirlos en derechistas, como privatización no debería significar concentrar más la riqueza de la nación en manos de la oligarqufa y de las empresas multinacionales sino que descentralización de la riqueza o democratización económica.

La época del cacique, caudillo, activista, agitador, burócrata, dirigentes vitalicios, campañas electorales de consignas, ya deberían haber pasado de moda en El Salvador, para dar paso al conocimiento, o sea a la capacidad intelectual, acompañada de la técnica y la ciencia, para resolver los problemas de la nación, mediante propuestas concretas y viables, asimismo construir canales ágiles y eficientes entre los partidos y la sociedad civil, donde se sientan y estén representados democráticamente en los órganos de dirección de tales partidos políti$\checkmark$ cos los campesinos, obreros, profesionales, intelectuales y miembros de la empresa privada, de vocación democrática y con identidad nacional.

Por otro lado, abordar el tema del bipartidismo en El Salvador es complicado cuando los partidos políticos se subdividen o atomizan, particularmente cuando están en la oposición. Esta es una de las razones por las cuales, no hay tradición partidaria ni ideológica entre un buen sector de la población.

Los partidos tienden a dar la impresión de monolíticos mientras detentan el poder político, porque éste puede mantenerlos unidos en la mayoría de los casos. Lo que daría la percepción entre algún sector de la población de que el partido más apto, capaz y de confianza para hacer gobierno es el partido oficial, a pesar de los desaciertos, comupción, autoritarismo, etc. De ser cierta esta hipótesis se - crea el serio problema de la alternabilidad del poder.

El autoritarismo militar y civil que se le ha impuesto a la nación a partir de la conquista de Cuscatlán ha cohibido el desarrollo de la democracia. Pero a partir de los acuerdos de paz firmados en 1992, se planteó la necesidad de que se iniciara un proceso de democratización y, por primera vez a partir de 1994, se permitió a los adversarios ideológicos, políticos y militares al régimen, tener la posibilidad de tener en la Asamblea Legislativa diputados miembros del FMLN. Sin embargo, en las elecciones para diputados y alcaldes celebradas el 10 de marzo de 1991, la coalición denominada Convergencia Democrática, fundada por los ex dirigentes del Frente Democrático Revolucionario (FDR), Guillermo Manuel Ungo, Héctor Oquelí y Rubén Zamora, entre otros, logró que se eligieran seis diputados en los 14 Departamentos y dos por la llamada plancha nacional24. 
- A partir de 1991 y principalmente desde 1994, la Asamblea Legislativa dej6 de ser un simple instrumento del Poder Ejecutivo.

La Asamblea está recuperando, su identidad propia, se está cambiando la imagen de "chivos" que tenía en el pasado el primer órgano del Estado al obedecer órdenes del Ejecutivo y el presidencialismo (sic) también irá cambiando, es un proceso, ya que "no se pueden cambiar de un plumazo doscientos años de historia"25.

Es cierto que la Asamblea tiende a cambiar su rol consuetudinario de ser un órgano decorativo, por haber estado constituido por diputados de derecha, que sin mayor análisis, estudio y discusión aprobaban cualquier proyecto de ley que enviara el Ejecutivo. Esto no significa que los diputados estén a salvo de la influencia y presión que ejercen el presidente y sus ministros, particularmente sobre el partido político oficial.

En teoría las decisiones que toman los diputados están en cierta medida influenciadas, en algunos casos, no sólo por sus respectivos partidos políticos, sino también por los grupos de presión, compadrazgos, etc.

Estas influencias indican si el partido es realmente democrático, en esa medida los diputados mantienen autonomía e independencia de los que buscan favorecer intereses minoritarios o de élite de cualquier clase y, por lo mismo, se inclinan por el interés de la nación.

Cuando a los diputados que se les impone la obediencia por parte de la dirigencia de su partido, significa que éste es antidemocráticó y que la unidad en la acción de los diputados con el Ejecutivo y la unidad orgánica que se manifiesta dentro de la fracción política en la Asamblea Legislativa, no es más que el resultado del autoritarismo que prevalece en el partido político y esto puede llevar a la conclusión de que es un partido fuerte por mostrar disciplina rígida, ya que ésta es el disfraz que encubre o esconde la sumisión incondicional a la autoridad de la cúpula del partido.

Aqui entra en conflicto la legitimidad y la representatividad, entonces a quién representa el diputado, a la cúpula de su partido o a sus electoreł. Asimismo se establece el conflicto entre legitimidad e ilegitimidad del rol del diputado.

- En general, si los partidos políticos son democráticos y, en particular, el partido oficial, entonces se tendrá una Asamblea Legislativa democrática, de lo contrario será autoritaria.

Según Gerson Martínez, jefe de la fracción legislativa del FMLN:

$\mathrm{Si}$ aquf las fracciones parlamentarias tuvieran autonomfa - todas- aqui hubiera más democracia parlamentaria. Lamentablemente las otras fracciones, especialmente la fracción del partido ARENA, es pretoriana, o sea verticalmente subordinada a la decisión del partido. Para ellos es inconcebible el 
voto diferenciado en su fracción. Ellos son unos soldados que reciben una orden y votan unánime como una plancha. Entonces no hay posibilidades en ellos de que fluyan opiniones distintas. Hay muchos ejemplos de eso.

Por ejemplo.

Cuando se estaba disculiendo sobre la industria del café, el jefe de la fracción de ARENA, Juan Duch, estuvo en el debate con posiciones parecidas a las nuestras: defendiendo una visión del sector cafetalero y el partido ARENA tenía otra posición, que era la oficial. Entonces el debate se dio, y dimos el debate: incluso Juan Duch dio el debate. A mi me sorprendió. Pero cuando dijeron "bueno, ahora se somete a votación", él votó igual que todos los dernás - miembros de ARENA - en contra de sus propios argumentos. Porque a la hora de votar ahí se aplica la espada de Damocles; a todos por igual.

Por eso yo creo que en la medida en que sean las fracciones pretorianas, o sea que reciban órdenes de arriba, entonces no va a haber verdadero parlamentarismo democrático en este pars26.

Este planteamiento puede ser refutado por los jefes de fracción de los demás partidos políticos, incluso por el de ARENA, para comenzar todos los institutos polfiticos en las Asamblea se autodenominan democráticos y de vocación popular, pero no es más que demagogia, porque una cosa es la que dicen y otra la que hacen, por ejemplo, como indicador podemos tomar las votaciones en las plenarias y en las sesiones de las comisiones legislativas, en las volaciones por regla general ARENA vota por leyes antipopulares y pro oligárquicas.

Es fácil saber identificar a los demagogos con sólo observar quienes votaron con ARENA en los siguientes:

La ley de apoyo a la reactivación del sector agropecuario (decreto 698) y por la ley de reestructuración de la deuda apraria (decreto 692 la cual consiste prácticamente en conducir al campesino a vender no sólo una parte de sus tierras, sino que a largo plazo lo condiciona a que venda toda sus tierras debido al ciclo del endeudamiento: con esta ley lo que se pretende es seguir favoreciendo al sector financiero del país.

Para aumentarle tres punlos al Impuesto del Val en propiedad de las asociaciones cooperativas, com unales y counitarias campesinas y beneficiarias de la reforma agraria (ecreto 719) con la cual prácticamente hacen desaparecer los escasos beneficios obtenidos de la llamada reforma agraria que surgió en 1980.

Por el decreto 707, con el cual los alcaldes gozarán de privilegios procesales, que no es otra cosa que otorgarles impunidad a la corrupción cometida por los alcaldes de ARENA.

Por la ley temporal de compensación económica por servicios prestados al Estado, conocida bajo el nombre de decreto 471, con la cual se dejaron 
desempleados a alrededor de 15 mil trabajadores del sector público, a la vez para tratar de desarticular y "descabezar" al movimiento sindical.

Por la ley transitoria de emergencia contra la delincuencia y el crimen organizado, la cual no sólo representa un retraso en los acuerdos de paz, sino que transgrede la Constitución Política de El Salvador, el ordenamiento jurídico interamericano y el derecho internacional público. -

En este mismo orden, los campesinos y obreros del campo miembros del Foro Nacional para la Defensa y Recuperación del Sector Agropecuario, se preguntan: “¿Hasta cuándo esta Asamblea Legislativa dejará de producir tantas leyes inconsultucionales? ¿Hasta cuándo los dipulados asumirán un papel consecuente con su discurso y defenderán la Constilución y constituirán un Estado de derecho?"27.

Ante estas leyes demagogicas y anticonstitucionales, los jefes de fraccion esgrimirán que sus partidos son democrálicos porque dejan en libertad a sus

- diputados para que voten como ellos lo consideren conveniente, pero únicamente en aspectos intrascendentes para la población. Al respecto el diputado Alejandro Dagoberto Martoquín, jefe de fracción legislativa del Partido de Conciliación Nacional (PCN), asegura que:

En cuestiones trascendentales en las que viene la línea del partido, ahi no nos podemos mover; pero en cuestiones, vaya, en correspondencia hay 30 piezas de correspondencia (sic) hombre hay 30 oportunidades, alguien no va a estar de acuerdo con alguien, verdad, entonces no vota; pero no trasciende28.

Esta situación autoritaria no se planteara si el partido fuera democrático y, por consiguiente, si la línea polf́tico-partidaria dentro de la fracción legislativa fuera general o abierta y no especifica o cerrada, de tal forma que al diputado no lo condicionara a votar incluso hasta en contra de sus electores y de su propia voluntad. El autoritarismo de los partidos políticos es una limitante para el desarrollo cualitativo del mismo diputado y un obstáculo a la construcción de la democracia en el pals. "El ser independiente de los partidos políticos y del presidente les permile a los legisladores procurar beneficios para sus propios votantes y servir a intereses sectoriales"29.

Ante el carácter autoritario de los partidos políticos, demás está plantearse la necesidad de realizar audiencias de la sociedad civil, para escuchar y analizar sus puntos de vista sobre determinados proyectos de ley, puesto que al diputado ya se le ha impuesto a priori la decisión por que o por quién votar o no, independientemente del grado en que le afecte y opine la población, a través de sus asociaciones, Organizaciones No Gubernamentales (ONG's), fundaciones, sindicatos, gremios, comunidades, etc.

Sin embargo, el presidente Armando Calderón Sol, en su discurso pronunciado en la Asamblea Legislativa, en ocasión de celebrar el segundo año de su 
gestión presidencial, expresó que la Asamblea es un lugar donde: " ... la democracia plural y representativa cobra su máxima expresión, porque la democracia consiste, fundamentalmente, en respetar y escuchar a los amigos y a los adversarios"30. En palses donde los partidos son democráticos los diputados llaman a los organismos, asociaciones, colegios profesionales, etc., de la sociedad civil para escuchar sus puntos de vista y tomarlos en cuenta al momento de elaborar el proyecto de ley, el dictamen y efectuar la votación en las comisiones o en el plenario. Asimismo, los diputados pueden votar en contra de la opinión de la dirigencia partidaria respectiva y coincidir en la votación incluso con los rivales partidarios e ideologicos ${ }^{31}$.

\subsection{El rol del partido y del legislador}

- La pregunta a formular es para qué se elige a los diputados, será para mejorar las condiciones económicas, políticas, sociales, culturales, etc., de la población, de sus cúpulas partidarias o para beneficio propio. Será que el diputado es el que pierde la noción de algunos términos o será la población la que los confunde, por ejemplo: cuando el diputado dice que lo que hace en la Asarnblea Legislativa lo hace para lograr el bienestar de la población o de la nación. Pero los términos población y nación qué realmente significan en "boca" del diputado.

Si el problema se reduce aparentemente a la semántica, significa que en el fondo, lo que ocurre es que el diputado ha dejado de ser el representante de sus electores, por lo que él se encuentra subordinado a otros intereses, pero no a los de la nación.

Es necesario aclarar que así como privatización no es lo mismo que modernización, tampoco población y nación son sinónimos de partido político, clase política, interés personal, ni oligarquía, etc.

- La conducta de los diputados se encuentra no sólo condicionada, entre otros, por la naturaleza del partido político al cual pertenece, sino también al tipo de sistema de gobierno que prevalece en la sociedad. No obstante; el diputado debe de responder primero por los intereses de sus electores, ésta es la calidad principal del diputado, ya que él en forma individual encama la representación de sus compatriotas y su respectivo partido encama colectivamente el interés de la nación. En conclusión, ante la disyuntiva de cuál interés es el que debe de primar en las decisiones de los diputados, se debe conceder prioridad al interés de la nación sobre el interés partidario.s

AquI no existe contradicción entre diputado y partido político, puesto que ambos roles son complementarios, el diputado es el representante de la población que lo eligió y el partido es más que un simple vínculo, es el intermediario entre el pueblo y el gobierno. Existe contradicción cuando el diputado o el partido polĺtico o ambos dejan de cumplir los roles establecidos. 
Habida cuenta, por consiguiente, de que los partidos políticos son los encargados de llevar a cabo tareas fundamentales de intermediación entre el régimen político y la sociedad, $y$ han devenido en elementos imprescindibles para el correcto funcionamiento de la acción de gobierno, su propia operatividad tiene mucho que ver con la gobernabilidad del sistema políti$\mathrm{co}^{32}$.

- Los partidos políticos y los diputados al dejar de cumplir su rol en mención, ponen en peligro el régimen de partidos políticos, porque pierden credibilidad y confianza ante la sociedad civil, de la misma manera, pueden afectar negativamente un proceso de democratización, construcción o reconstrucción democrática o a la democracia misma, cuando ésta ya está institucionalizada.

\subsubsection{Diputados representantes de los ciudadanos}

- Los diputados de los diferentes partidos politicos representan en su conjunto al pueblo, según la Constitución Política de El Salvador, Art.25:"los diputados representan al pueblo entero". Art.85: "el sistema político es pluralista y se expresa por medio de los partidos polfticos, que son el único instrumento para el ejercicio de la representación del pueblo dentro del Gobierno"? De tal forma, que la Asamblea Legislativa representa institucionalmente a los ciudadanos y es aquí donde reside la soberania popular.e

Representación viene de representar, verbo que en el teatro o en el Derecho privado designa el hacer uno el papel del otro. Del latín praesse (que es un derivado del verbo esse, ser, el verbo clásico), es decir «poner delante» «mostran, gira en torno a esa semántica fundamental, en todas sus acepciones: representar un drama, representar unos agravios, representar a una sociedad, etc. ${ }^{33}$ En el orden jurídico, ese significado básico se matiza con el de la validez de los actos hechos a nombre de otro...34.

Según este concepto se puede inferir que cuando un diputado ha dejado de representar los intereses de sus conciudadanos, reemplazándolos por los partidarios, personales o de algún país extranjero, etc., entonces el diputado ha perdido legitimidad ante la nación y el carácter de representante de los electores, por lo que sus actos carecen de validez.

“...El legislador es representativo por responder ante los problemas legislativos, de igual modo en su conjunto que lo harían sus coterráneos en el distrito electoral" 35 , dentro de esta lógica la población a través del rol de representación con el cual invisten a los diputados, ejerce su voluntad soberana, influencia y control sobre el gobierno. Sin embargo, los diputados al dejar de cumplir la función que les fue delegada a través del sufragio universal, a fectan también el equilibrio de poderes del Estado y ponen en peligro la gobernabilidad del pafs. En este sentido, el divorcio entre el partido político y el pueblo produce 
abstencionismo electoral, o sea una pérdida de confianza en el poder del voto. Los dirigentes de los partidos políticos cuando están en campaña electoral se presentan como la mejor opción y única alternativa para resolver los problemas que más afectan a la población, pero cuando éstos son elegidos no cumplen lo que prometieron.

Los ciudadanos no solo se abstienen de hacer uso del sufragio, sino que, además, se retiran o dejan de tener militancia activa en los partidos polf́ticos al sentirse defraudados y desencantados, la sociedad civil se da cuenta que únicamente es instrumentalizada para que los políticos y sus respectivos partidos obtengan beneficios, esta siluación conduce a una crisis de credibilidad en los partidos políticos.

Los representantes por los que votamos merecen nuestro apoyo por que llenan uno o más de los siguientes requisitos: actúan como portavoces de nuestra ideologfa y visión de la forma correcta de gobierno; procuran el bienestar de nuestro territorio (pueblo, ciudad, región, etc.), y posibilitan la sobrevivencia y el mejoramiento de una minorfa étnica.El buen sistema electoral es el que establece un vínculo apropiado entre delegación y representación. El mal sistema, por el contrario, produce injusticia electoral, crea en el elector un sentimiento de distancia y futilidad e induce al ciudadano a creer que votar no vale la pena. De estos pareceres dan cuenta las tasas de abstencionismo y papeletas nulas ${ }^{36}$.

Según una encuesta realizada en febrero de 1995, por el Instituto de Opinión Pública (IUDOP) de la Universidad Centroamericana "José Simeón Cañas"

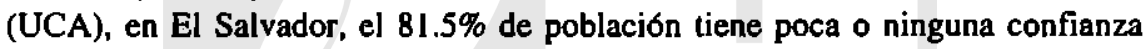
en los partidos políticos37. Este porcentaje no se traduce s6lo en una crisis de credibilidad, sino que también en una crisis de representalividad y es un fenómeno que afecta a los partidos políticos como instituciones, asimismo a los políticos tanto de la derecha como de la izquierda. El dirigente sindical José Guadalupe Martínez, presidente de la Central de Trabajadores Democráticos de El Salvador (CTD), considera que: "los diputados del FMLN no están cumpliendo su papel de trabajar en la Asamblea Legislativa en beneficio del pueblo y califico su desempeño como «nulo»"38.

Se percibe que la población tiene una concepción negativa de la política, debido a la conducta y comportamiento de los políticos y del papel oportunista y demagógico de sus respectivos partidos, a tal grado, que la connotación de ciencia y arte de la política, ha sido sustituida en El Salvador por ciertos calificativos, entre ellos podemos mencionar los siguientes: "chuca", sucia, traidora, compla, impune, autoritaria, sinvergüenza, traficante, mentirosa.

Esta concepción negativa se agrava aún más con el bajísimo vocabulario que emplean algunos políticos y debido a los ataques verbales entre ellos y contra la población salvadoreña, el léxico de los políticos llega a rimar incluso hasta con 
lo vulgar e inmoral.

Es necesario evitar actitudes que denotan una falta de cultura política del diputado, sobre todo en los debates irrespetuosos o las críticas personales que lesionan la integridad moral de sus contendientes y obligan a la replica en similares términos. Esto presupone la necesidad de elevar el nivel del debate político, fomentar la autoestima y practicar el respeto mutuo entre todos los diputados 39 .

A manera de ejemplo vamos a mencionar solamente un reducido número de términos y expresiones utilizados recientemente por algunos políticos:

- Juan José Daboud, presidente de la Administración Nacional de Telecomunicaciones (ANTEL): "No, me referi al modelo estatal que consume recursos y DEFECA una serie de problemas" 40.

- Eduardo Interiano, ministro de salud pública y asistencia social, califica y además les "receta" a las mujeres salvadoreñas que le solicitan promover un diálogo y buscar soluciones sobre el aborto: "Debemos decirle también que no nos parece de la altura de un Ministro la repuesta de calificar como LOCAS y recetar "ELECTRO-SHOCKS" a quienes opinamos en este sentido"4l.

- Julio Valdivieso, viceministro del transporte, ante el paro temporal de transporte convocado por la Asociación de Empresarios de Transporte de Pasajeros (ATP), a principios del año, el viceministro: "calificó a los miembros de ATP de ANTISOCIALES" 42 .

- Mario Beltaglio, gobernador político del Departamento de San Miguel, al referirse a Mabel Quintanilla, ex sargento de la Policfa Nacional Civil (PNC), lo hizo de la siguiente manera: "yo no voy a ponerme a un dime que te diré con una PROSTITUTA"43.

- Alejandro Dagoberto Marroquín, diputado por el Departamento de San Salvador, jefe de fracción legislativa del Partido de Conciliación Nacional (PCN), miembro de las comisiones legislativas, política, de hacienda y especial del presupuesto, de relaciones exteriores e Integración centroamericana y del comité lécnico, dice: "... YO NUNCA HABLO PAJA..." 44.

- Norman Noel Quijano, diputado por el Departamento de San Salvador por el partido ARENA, miembro de las comisiones legislativas de trabajo y previsión social y de protección al medio ambiente y salud pública: “...sólo que sea BRUTO no aprende hablar... es bien fácil hablar el MONTÓN DE PAJA por diez minutos... sólo que sea TAN BRUTO que no se le quede..."45.

- Walter René Araujo Morales, diputado por la circunscripción nacional por el partido ARENA, secretario de la junta directiva, presidente de la comisión de 
legislación y puntos constitucionales y miembro de la comisión política de la Asamblea Legislativa, calificó a los autores del comunicado de la Fuerza Nacionalista "Mayor Roberto D' Abuisson" (FURORA), como: "CERDOS e IMBÉCILES"46.

- Arturo Argumedo h., diputado por la circunscripción nacional miembro del Partido Demócrata Cristiano (PDC), secretario de la comisión de legislación y puntos constitucionales y miembro de la comisión política de la Asamblea Legislativa: "Ha llegado la hora de las confesiones ME CONFIESO ALCOHÓLICO"47.

- Joaquín Villalobos, ex-comandante del FMLN, actualmente es alto dirigente del Partido Demócrata (PD), al referirse a la inhabilitación política por diez años que recomendó el informe de la Comisión de la Verdad, lo hace en los siguientes términos: "La inhabilitación política fue una ESTUPDEZ"48.

- Eduardo Sancho, ex-comandante del FMLN, actualmente es alto dirigente del PD y, secretario de la junta directiva y miembro de la comisión política de la Asamblea Legislativa, él denomina al dirigente político del Partido Liberal Democrático, así: "Kirio Salgado es un MEDIOCRE FRUSTRADO que sólo busca el oportunismo"49.

- Juan Duch Martínez, ex-alcalde de Santa Ana, actualmente diputado por el Departamento de Santa Ana, jefe de fracción legislativa de ARENA y, miembro de las comisiones política y de hacienda y especial de presupuesto y la Srita. Mercedes Gloria Salguero Gross, diputada por la circunscripción nacional por el partido ARENA, presidenta de la Asamblea Legislativa y presidenta de la comisión política de la Asamblea. "Juan Duch y Gloria Salguero Gross calificaron de TERRORISTAS E INGRATOS a los diputados del FMLN que no asistieron a la ceremonia del segundo aniversario de la gestión de Calderón Sol"'50.

- Armando Calderón Sol, presidente de El Salvador, se refirió a los salvadoreños, que critican la ley transiloria de emergencia contra la delincuencia y el crimen organizado, por violar la Constitución Política de El Salvador, el sistema jurídico interamericano y el derecho internacional público, con los siguientes términos: "... una SUCIEDAD POLfTICA, porque hay muchos CERDOS POLf́TICOS"'si. De igual forma, "el presidente calificó de MAL NACIDOS y SEUDO IZQUIERDISTAS a los sindicalistas y diputados de la oposición que denunciaron en el extranjero las violaciones al derecho laboral en las empresas maquiladoras..." 52.

El bajo nivel alcanzado por la clase política y sus respectivos partidos, es una de las causas fundamentales para que los ciudadanos se abstengan de emitir el sufragio o que anulen el voto el día de las elecciones e impide que militen en los partidos políticos, porque eso serfa para una buena parte de salvadoreños 
degradarse o rebajarse al nivel de los políticos.

\section{NOTAS Y REFERENCIAS}

1. Real Academia Española, Diccionario de la Lengua Española, Madrid, Real Academia Española, Vigésima Edición, Tom.Il, 1986, p.1015.

CFR, Paul Rober, Le petit Robert Dictionnaire alphabétique et analogique de la langue française, Paris, Le Robert, 1985, p.1361.

2. Ramón García Cotarelo y Andrés de Blas Guerrero, Teoría del Estado y Sistemas Políticos, Madrid, Universidad Nacional de Educación a Distancia, Tom.l, 1978, p.351.

3. Monstesquieu, Del espíritu de las leyes, México, Porrúa, 1977, p. 104.

4. Rogelio Hernández, El Congreso Mexicano, México, Instituto de Investigaciones Legislativas, 1994, pp.190-191.

5. Nicolás Pérez Serrano, Tratado de Derecho Político, Madrid, CIVITAS, Segunda Edición, 1984, p.821.

6. E. Allan Farnsworth, Introduction au système juridique des Etats-Unis, Paris, Nouveaux Horizons, 1976, p.99.

7. Vide, Kaye Holloway, Le Canada pourquoi l'impasse?, Montreal, Editions Nouvelle Optique, Tom.I, 1983, p.120.

8. Ivo D. Duchacek, Mapas del poder, político constitucional comparada, Madrid, Instituto de Estudios Polfticos, 1976, p.289.

9. Ibidern, p.300.

10. Juan Jacobo Rousseau, El Contrato Social, San José, Editorial Universitaria Centroamericana (EDUCA), Tercera edición, 1980, p.67.

11. Winfried Staffani, "Sobre la distinción entre los sistemas de gobiemo parlamentario y presidencialista", Democracia representativa y parlamentarismo, Buenos Aires, Centro Interdisciplinario de Estudios sobre el Desarrollo Latinoamericano (CIEDLA) y la Konrad-Adenauer-Stiftung, 1984, p.17.

12. CFR, Alberto Zarza Menzaque, "Presidencialismo y parlamentarismo", Documentos y conclusiones del primer congreso internacional de modernización parlamentarla, Buenos Aires, Honorable Congreso de la Nación Argentina, 1990, p.228. CFR, Enrique Tarigo, "Presidencialismo y parlamentarismo en la evolución constitucional uruguaya", Documentos y conclusiones del primer congreso internacional de modernización parlamentaria, Ed. cit. p.99.

13. Mario Verdugo Marinkovic y Ana Marla Barzellato, Manual de derecho político. Santiago de Chile, Editorial Jurídica de Chile, Tom.I, 1988, p.223.

14. El nombre del jefe de gobiemo, varía la denominación dependiendo del país, por ejemplo, en algunos es llamado, Canciller, Presidente del Consejo, Premier, Primer Ministro, etc.

El jefe de Estado en las monarqulas constitucionales parlamentarias, es un puesto hereditario y lo ocupa el Rey o la Reina, por ejemplo: Reino Unido, Suecia, Noruega, Dinamarca, España, Bélgica, etc. En los Estados parlamentarios republicanos por lo general lo es el presidente, por ejemplo: Francia, Italia, Alemania, etc.

15. Ley Fundamental de Bonn (1949), art.67.

16. Constitución Española (1978), art.113.2 y art.114.2.

17. Delia Ferreira Rubio y Matteo Goretti, "La reforma constitucional Argentina: ¿un 
presidente menos poderoso?", Contribuciones, Buenos Aires, Centro Interdisciplinario de Estudios sobre el Desarrollo Latinoamericano (CIEDLA) y la Konrad-Adenauer-Stiftung, No.1, enero-marzo de 1995, p.73.

18. Mario Verdugo Marinkovic y Ana Mara Barzellato, Ed. cit., p.227.

19. Linz, J., "Los peligros del presidencialismo", Reformas al presidencialismo en América Laúna, Comisión Andina de Juristas, Caracas, 1993, p.31.

20. Nohlen, D., "Sistemas de gobierno, perspectivas conceptuales y comparativas", Reformas al presidencialismo en América Latina, Ed. cil., passim.

21. Carlota Jackisch, "Algunos aspectos del debate actual sobre presidencialismo vs. parlamentarismo en Latinoamérica, Contribuciones, Ed. ciL, No.1, enero-marzo de 1995, p.62.

22. Carlos Sabino. "El sistema polftico venezolano: estabilidad, crisis e incertidumbre", Contribuciones, Ed. cit., No.1, enero-marzo de 1995, p.161.

23. Vide, Frente Farabundo Martí para la LiberaCión Nacional, El plan del Frente para lograr una economía productiva con desarrollo humano, San Salvador, FMLN, abril de 1996.

Aquiles Montoya, "A propósito de la propuesta del FMLN", ECA Estudios Centroamericanos, San Salvador, Universidad Centroamericana "José Simeón Cañas" (UCA), No.570, abril de 1996, p.358.

24. CFR, Carlos Acevedo, "El significado político de las eleceiones del 10 de marzo", ECA Estudios Centraamericanos, Ed. cil., No.509, marzo de 1991, p.158.

"Diputados electos: 10 de marzo 1991", Revista de la Universidad Tecnológica, San Salvador, UTEC, Año.2, No.3, marzo, abril y mayo de 1991, p.112.

25. "Debate sobre la labor de Comisión Polftica: Asamblea ya no recibe ordenes del Ejecutivo", La Prensa Gráfica, (San Salvador), 27-12-1994, p.8A.

26. Entrevista realizada al diputado Gerson Martínez jefe de fracción del FMLN, el día 25-07-1995, a las 11:15 A.M., en la oficina del jefe de fracción en la Asamblea Legislativa

27. Foro Nacional para la defensa y recuperación del sector agropecuario, "Tribuna Agropecuaria", Co Latino, (San Salvador), 20-06-1996, p.8.

28. Entrevista realizada al diputado Alejandro Dagoberto Marroquín jefe de fracción del PCN, el día 20-07-1995, a las 10:04 A.M., en el local de la fracción del PCN en la Asamblea Legislativa.

29. James A. Thurber, "Introducción: las raices de la democracia dividida", La democracie dividida, Buenos Aires, Editorial Heliastas, 1995, p.17.

30. Armando Calderón Sol, "Discurso pronunciado por el señor presidente de la República doctor Armando Calderón Sol, en ocasión de celebrar el segundo afío de su gestión presidencial", La Prensa Gráfica, (San Salvador), 03-06-1996, p.38A.

31. Ramón Garcla Cotarelo y Andrés de Blas Guerrero, Op. cit., p.47.

32. Manuel Alcántara Saez, "Partidos politicos y gobernabilidad", Espacios Revista Centroamericana de Cultura Política, San José, Facultad Latinoamericana de Ciencias Sociales (FLACSO), No.3, enero-marzo de 1995, p.5.

Vide, Stefan Roggenbuck, "Instituciones y democtacia en América Latina", La Prensa Grafica, (San Salvador), 25-11-1996, p.8A.

Vide, Stefan Roggenbuck, "Instituciones y consolidación democrática en Latinoamérica", Instituciones y democracia en El Salvador, San Salvador, Fundación Ronrad Adnauer Stiftung, 1994, p.6. 
33. CFR, Rodrigo Femández Carvajal, La representación pública en la actualidad, S.l.i., Murcia, 1965, passim.

34. Manuel Fraga Iribane, Legitimidad y representación, Barcelona, Grijalbo, 1973, p.169.

35. Carl J. Friedrich, Gobiemo constitucional y democracia, Madrid, Instituto de Estudios Políticos, 1975, p.117.

36. Carlos Granados Ch., "Territorialidad y justicia electoral en Costa Rica", Revista Parlamentaria, San José, Asamblea Legislativa de Costa Rica, Vol.2, No.2, diciembre de 1994, p.215.

37. Instituto Universitario de Opinión Pública, La opinión de los salvadoreños sobre la situación política del país, San Salvador, IUDOP, Boletĺn de prensa Año.X, No.2, febrero de 1995, p.4.

Vide, Fundación para la investigación del desarollo humano en El Salvador, "Carta de fundación", Diario Latino, (San Salvador), 28-09-1995, p.5.

38. Oscar Dlaz y Tania Moreno, "Trabajadores critican a diputados del FMLN", La Prensa Gráfica, (San Salvador), 12-11-1996, p.7A.

39. Asamblea Legislativa, "Madurez democrática: conclusiones del seminario «imagen del diputado", La Prensa Gráfica, (San Salvador), 30-04-1996, p.8C.

40. "Presidente de ANTEL se retracta", Co Latino, (San Salvador), 17-04-1996, p.3.

41. Asociación de madres demandantes por la cuota alimenticia, IMU, Las Dignas y MAM, "Carta al Señor Ministro de Salud Pública y Asistencia Social, Dr. Eduardo Interiano", La Prensa Grálica, (San Salvador), Asociación de Madres Demandantes por la Cuota Alimenticia, Instituto de la Mujer (IMU), Mujeres por la Dignidad y la Vida (Las Dignas) y el Movimiento de Mujeres Mélida Anaya Montes (MAN), 27-07-1996, 43A.

42. "Miles de usuarios caminan por paro de buses", La Prensa Gráfica, (San Salvador), 23-02-1996, p.3A.

43. Liliana Fuentes Monroy, "Bettaglio rechaza informe", La Prensa Gráfice, (San Salvador), 14-03-1996, p.50A.

44. Entrevista realizada al diputado Alejandro Dagoberto Marroquín jefe de fracción del PCN, el dia 20-07-1995, a las 10:04 A.M., en el local de la fracción del PCN en la Asamblea Legislativa.

45. Entrevista realizada a Norman Noel Quijano, diputado de ARENA, Relator de la Comisión de trabajo y previsión social, el dia 07-09-1995, a la 1:45 P.M., en el local de la fracción de ARENA en la Asamblea Legislativa.

46. Nelson Dueñas, "APES preocupada por amenazas", Co Latino, (San Salvador), 27-06-1996, p.24.

47 "Caza de citas", Primera Plana, (San Salvador), Año.1, No.35, del viemes 26 de mayo al 1 de junio de 1995, p.2.

48. "Caza de citas", Primera Plana, (San Salvador), Año.1, No.32, del viemes 5 al 11 de mayo de 1995, p.2.

49. "Caza de citas", Primera Plana, (San Salvador), Año.1, No.17, del viemes 13 al 19 de enero de 1995, p.2.

50. "Los del FMLN son unos ingratos, inadaptados", Diarlo de Hoy, (San Salvador), 03-06-1996, p.15.

51. Marfa Pérez, "Calderón llama «cerdos políticos» a los que se oponen al fuero edilicio", Co Latino, (San Salvador), 23-05-1996, p.3. 
Vide, “¿habrá voluntad para hacer pactos?”, La Prensa Gráfica, (San Salvador), 31-05-1996, p.5A.

52. "Crónica del mes: noviembre-diciembre", ECA Estudios Centronmericanos, Ed. cit., No.565-566, noviembre-diciembre de 1995, p.1173.

Alfredo Hernández, "Malnacidos quienes hacen falsas denuncias en maquilas", La Prensa Gráfica, (San Salvador), 29-11-1995, p.5A. 